\title{
Experiencias y retos del Centro de Apoyo Técnico en la investigación integrada en los servicios en América Latina y el Caribe
}

\author{
Víctor Becerril-Montekio ${ }^{1}$, Pilar Torres-Pereda ${ }^{1}$, Luis Alberto García-Bello ${ }^{1}$ y Jacqueline Alcalde-Rabanal'
}

Forma de citar Becerril-Montekio V, Torres-Pereda P, García-Bello LA, Alcalde-Rabanal J. Experiencias y retos del Centro de Apoyo Técnico en la investigación integrada en los servicios en América Latina y el Caribe. Rev Panam Salud Publica. 2021;45:e19.

https://doi.org/10.26633/RPSP.2021.19

RESUMEN

En este artículo se describen los principales modelos de integración, las experiencias de éxito y los retos del trabajo conjunto de los investigadores y los tomadores de decisiones participantes en la iniciativa Incorporación de la Investigación para Avanzar en el Cumplimiento de los Objetivos de Desarrollo Sostenible (ER-SDG), y la experiencia del Centro de Apoyo Técnico (CAT). En junio de 2018 se otorgó financiamiento, previa selección, a 13 proyectos de investigación de 11 países de ingresos medios y bajos de América Latina y el Caribe (Argentina, Bolivia, Brasil, Colombia, Ecuador, Guatemala, Guyana, Haití, Paraguay, Perú y República Dominicana). Los proyectos debían estar centrados en los cambios que se requieren a nivel de sistema, políticas o programas para mejorar la salud y basarse en el trabajo conjunto de investigadores y tomadores de decisiones, a fin de acercar la generación de evidencias a la toma de decisiones en los sistemas y servicios de salud. EI CAT apoyó y orientó la producción de resultados de calidad y de utilidad para la toma de decisiones. La experiencia confirmó el valor de iniciativas como ER-SDG en la consolidación de puentes entre el mundo de la investigación sobre implementación de políticas, programas y sistemas de salud, y el mundo de los funcionarios encargados de operar esos programas, servicios e intervenciones relacionadas con la salud. Se resalta la necesidad de respetar y aprovechar cada contexto, y los arreglos y patrones específicos de relación entre investigadores y tomadores de decisiones, mediante incentivos para la integración.

Palabras clave Ciencia de la implementación; política informada por la evidencia; investigación sobre servicios de salud; América Latina.

Entre los esfuerzos para acercar la generación de resultados científicos validados a la toma de decisiones en los sistemas y servicios de salud se destaca la iniciativa Mejora de la Implementación de Programas a Través de la Incorporación de la Investigación (iPIER, por las siglas en inglés de Improving Program Implementation through Embedded Research) (1). Esta iniciativa, que estuvo patrocinada conjuntamente por la Organización Panamericana de la Salud (OPS) y la Alianza para la
Investigación en Políticas y Sistemas de Salud (AHPSR), tuvo dos ediciones: una en 2014 y otra en 2016.

Partiendo del llamado de la Organización Mundial de la Salud (OMS) para "integrar la investigación en los servicios de salud" (2), esta iniciativa estimulaba que los implementadores de programas y políticas de salud de América Latina y el Caribe diseñaran y lideraran investigaciones científicas pertinentes y de calidad. Además de mejorar la ejecución y el funcionamiento

\footnotetext{
1 Centro de Investigación en Sistemas de Salud, Instituto Nacional de Salud Pública,

Cuernavaca, Morelos, México. $₫$ Pilar Torres-Pereda, pilar.torres@insp.mx
} 
de intervenciones, servicios y programas de salud, procuraba acercar la investigación a la toma de decisiones y, al mismo tiempo, vencer importantes retos en torno a la forma de lograrlo, aspectos sobre los que es necesario reflexionar.

La iniciativa iPIER se diseñó para promover la investigación sobre la implementación integrada en los servicios mediante la combinación de las capacidades, la experiencia y el conocimiento del contexto del personal de salud que opera los programas, con el conocimiento teórico y la experiencia práctica de investigadores. Por ello, los equipos seleccionados para participar debían estar integrados por el tomador de decisiones responsable de alguna política, un programa o ciertos servicios de salud —quien asume el papel de líder o investigador principal-y por investigadores a tiempo completo pertenecientes a la misma institución de salud, una institución académica vinculada con la primera o alguna asociación civil con experiencia en investigaciones relacionadas con la salud.

En 2018 se presentó una iniciativa similar, denominada Incorporación de la Investigación para Avanzar en el Cumplimiento de los Objetivos de Desarrollo Sostenible (ER-SDG, por las siglas de su nombre en inglés Embedding Research for the Sustainable Development Goals), patrocinada por la OPS, la AHPSR y el Programa Especial de Investigación y Capacitación en Enfermedades Tropicales, de la OMS (TDR/OMS) (3). Esta iniciativa comenzó con una convocatoria abierta en la que se invitaba a tomadores de decisiones de países de ingresos medios y bajos de América Latina y el Caribe a enviar propuestas de investigación centradas en los cambios que se requieren a nivel de sistema, políticas o programas para mejorar la salud. Como en el caso de la iniciativa iPIER, el enfoque de partida aquí era colocar al responsable de las decisiones - o encargado de ejecutarlas- en la posición de investigador principal jefe del proyecto de investigación.

Dado el carácter innovador de la investigación sobre implementación y de su integración dentro de los servicios de salud (4-8), se creó el Centro de Apoyo Técnico (CAT), formado por investigadores con experiencia probada, para brindar apoyo técnico. Entre sus metas estaban asegurar la comprensión de los objetivos y la metodología de la investigación sobre implementación, contribuir al mejor aprovechamiento de los resultados, asesorar en la elaboración de recomendaciones basadas en datos científicamente comprobados y apoyar en la generación de materiales —como resúmenes ejecutivos e informes técnicos sobre políticas (policy briefs) — que ayudaran a sustentar la toma de decisiones. La iniciativa ER-SDG fortalecería el proceso de integración de la investigación dentro de los servicios, los programas y las políticas de salud, lo que fomentaría la discusión y la colaboración entre los investigadores y el personal de salud. Como premisa, todo ello se realizaría sobre la base del respeto irrestricto a la conformación particular de cada equipo, la autonomía y el liderazgo de los responsables de cada proyecto.

En este artículo — cuyos autores formaron parte del CAT— se describen los principales modelos de integración, las experiencias de éxito y los retos del trabajo conjunto de los investigadores y los tomadores de decisiones participantes en el ER-SDG.

Entre abril y mayo de 2018 se recibieron más de 200 propuestas de investigación (en forma de notas conceptuales) que fueron rigurosamente analizadas por las instituciones patrocinadoras según los siguientes criterios: a) objetivo claro, articulado en términos de cómo se puede mejorar un programa, un sistema o una política de salud, o corregir sus fallas, mediante la investigación de esos programas, sistemas y políticas; b) evidencia demostrada de compromiso efectivo para apoyar la toma de decisiones informadas a partir de resultados científicamente validados; c) apoyo y preparación de la(s) institución(es) para garantizar la implementación exitosa del proyecto; d) demostración de una sólida comprensión técnica del tema propuesto; e) capacidad para llevar a cabo investigaciones sobre políticas y sistemas de salud; y f) disposición para usar los resultados en la mejora de las políticas, los sistemas o los programas (3).

En junio de ese mismo año, la OPS, la AHPSR y el TDR/OMS seleccionaron y otorgaron financiamiento a 13 equipos de 11 países de ingresos medios y bajos de América Latina y el Caribe en los que se habla francés (Haití), inglés (Guyana), portugués (Brasil) y español (Argentina, Bolivia, Colombia, Ecuador, Guatemala, Paraguay, Perú y República Dominicana); participaron dos equipos de Colombia y dos de Guatemala. A pesar de la diversidad de lenguas y culturas de los países participantes, sus realidades socioeconómicas y sociopolíticas eran similares; aun así, las diferentes problemáticas tratadas obligaron a hacer un esfuerzo adicional para aprovechar tanto las similitudes como las diferencias de estos equipos de trabajo y contextos. Los equipos mostraban diversos grados de experiencia en investigación, relaciones nuevas o de larga data entre los tomadores de decisiones y los investigadores, así como formas de organización institucional más o menos favorables para la integración de la investigación en los servicios de salud. En tan disímiles condiciones, se debió encontrar la mejor adaptación para poder descubrir las fallas de alguna estrategia de implementación, las barreras existentes en el sistema de salud, y conclusiones útiles para sustentar la mejor toma de decisiones y proponer mejoras razonables y factibles.

El CAT apoyó y orientó la producción de resultados de calidad y de utilidad para la toma de decisiones mediante las siguientes acciones: taller presencial de desarrollo de protocolo de investigación y taller presencial de análisis de datos y traducción de conocimiento; reuniones y llamadas periódicas de seguimiento; seminarios virtuales sincrónicos y asincrónicos; entrevistas formales; y bitácoras de seguimiento. Durante todo el proceso y al final se realizaron lecturas y análisis puntuales de los resultados de investigación de cada uno de los equipos para poder orientarlos y apoyarlos en el desarrollo de investigaciones sobre implementación integrada en los servicios, que cumplieran con los más altos estándares de calidad científica.

Se encontraron varias modalidades o patrones básicos de relación entre tomadores de decisiones e investigadores. El punto de partida fue el concepto de integración (embeddedness) de la investigación en los servicios de salud, en el sentido de la participación directa de los tomadores de decisiones y el personal de salud en el proceso mismo de la investigación (7). En los 13 equipos participantes se observaron diversas modalidades, patrones y grados de integración de la investigación, por lo que el equipo del CAT debió adecuarse respetuosamente a las posibilidades y los contextos de cada uno de ellos para apoyarlos en su objetivo de lograr el mayor beneficio en términos de mejoras de su sistema de salud.

Los patrones de integración se expresaron en el tipo de relación desarrollada entre los implementadores de los programas o servicios de salud y los investigadores para proponer, desarrollar y llevar a buen término el proyecto de investigación que propusieron en el marco de la iniciativa ER-SDG. En todos los casos, se respetó la conformación de los equipos seleccionados, 
y se reconoció y adecuó la actividad del equipo del CAT a tres modalidades o patrones generales de colaboración entre los tomadores de decisiones y los investigadores: a) básicamente formales, b) de aprendizaje mutuo y c) de consolidación de la colaboración.

Relaciones básicamente formales. Se encontraron propuestas de investigación sobre implementación integrada en los servicios de salud en los que predominaba el equipo de investigadores, generalmente pertenecientes a instituciones académicas, con un evidente conocimiento teórico y experiencia práctica, en cuyo criterio los tomadores de decisiones depositaron su entera confianza. En términos generales, el personal de investigación definió, diseñó y ejecutó la propuesta de investigación contando con el acompañamiento y el apoyo logístico, administrativo e institucional de quienes toman las decisiones en los servicios de salud. Sobre estas bases, el conocimiento, la experiencia y el profesionalismo de los investigadores garantizaron la calidad de la investigación, mientras la confianza mutua abrió la vía para la utilización de los resultados de la investigación. Se pudo constatar que esta modalidad de colaboración fue exitosa y generó resultados de investigación de alta calidad científica sobre cuya base se logró elaborar recomendaciones, resúmenes ejecutivos e informes técnicos de políticas de salud. Sin embargo, se debe reconocer como principal limitante que este tipo de colaboración no incide directamente en el fortalecimiento de capacidades de investigación de quienes toman las decisiones y en la integración de la investigación en los servicios $y$, aunque puede dirigir sus esfuerzos a la escritura de artículos científicos, no necesariamente produce el objetivo esperado: la mejora de los programas. En otras palabras, este tipo de relación no favorece que el personal de salud amplíe sus capacidades de generación de resultados validados científicamente y, por tanto, que se perciban a sí mismos como coautores directos y copropietarios de los resultados de la investigación. La participación del CAT — al igual que las relaciones entre los tomadores de decisiones y los investigadores locales- permaneció en un nivel de cordialidad formal que propició intercambios satisfactorios de productos de buena calidad. Sin embargo, con este modelo no se logró reforzar significativamente las capacidades investigativas de los actores directamente involucrados en los servicios y los programas de salud.

Relaciones de aprendizaje mutuo. En este caso, la relación inicial entre los dos equipos es similar a la anterior: los tomadores de decisiones y los investigadores confían unos en otros y existe un claro acuerdo para el trabajo colaborativo. No obstante, el involucramiento de los tomadores de decisiones es más activo y directo, tanto en el desarrollo del protocolo de la investigación como en su ejecución y el análisis de los resultados. En ocasiones, esto se originó por una mayor experiencia en investigación de los tomadores de decisiones y, en otras, se apoyó o se combinó con una mayor disposición de los investigadores a propiciar la intervención directa de los primeros en el proceso investigativo. De esta manera, los tomadores de decisiones se volvieron copartícipes de un proceso de aprendizaje mutuo, en el que, al tiempo que aportaron su conocimiento del terreno, fortalecieron su capacidad de análisis. Las bases de confianza se reforzaron, el personal de salud se benefició de su acercamiento directo al proceso de investigación y comprendió mejor el carácter y el alcance de los productos que se generaron, mientras quienes normalmente investigaban desde fuera de los servicios, accedieron a una visión más cercana de su funcionamiento en el terreno. En estos casos, se constató una influencia positiva de la participación del CAT como facilitador de la relación entre investigadores y tomadores de decisiones, al propiciar la comunicación mediante llamadas, correos electrónicos y seminarios en línea. Al final del proceso de investigación, ambas partes compartieron la propiedad de los resultados, lo cual no solo abre la posibilidad de su utilización, sino que implica un fortalecimiento de los implementadores en investigación en general como fuente de resultados científicamente validados que sustenten la toma de decisiones y generen la posibilidad de utilizar resultados científicos de diferente índole en su proceso de toma de decisiones.

Este esquema es, sin dudas, el entorno ideal de la iniciativa ER-SDG, dado que al tiempo que se fortalecían las capacidades de investigación de las personas dentro del sistema de salud, se generaron datos confiables que responden a necesidades del sistema. No obstante, existe el riesgo de que el sesgo de las personas que desarrollan las intervenciones incline la balanza hacia las bondades de sus programas más que hacia sus deficiencias. Ante ello, la presencia de un actor neutral — posición que ocupó el CAT - ofrece una interlocución valiosa que permite a los actores adoptar posiciones más objetivas.

Relaciones de consolidación de la colaboración. En estos casos, las personas que tomaban las decisiones dentro del sistema de salud y las que hacían investigación de manera habitual solían tener antecedentes importantes de colaboración. Cuando existen arreglos institucionales adecuados, puede que los investigadores ya laboren dentro de las instituciones de salud. También es posible que, aun perteneciendo a instituciones académicas, hayan desarrollado un vínculo de colaboración gracias a investigaciones anteriores. Iniciativas como ER-SDG ofrecen condiciones ideales para consolidar esta colaboración, al garantizar un espacio de financiamiento, aprendizaje y acción para la mejora en la toma de decisiones. El aprendizaje de la metodología de investigación sobre implementación integrada en los servicios, novedosa para ambas partes, reforzó tanto la horizontalidad preexistente en la relación de colaboración como la propiedad compartida de los resultados de la investigación. Al igual que en el modelo anterior, las actividades promovidas por el CAT facilitaron el contacto y la comunicación entre los actores involucrados en la generación de esos resultados.

En casos en los que las relaciones datan de mucho tiempo atrás, las fallas suelen obedecer a relaciones de poder previamente establecidas. También aquí, la presencia de un actor independiente — como el CAT — puede apoyar, al ofrecer una visión neutral de los procedimientos en apego al rigor científico.

En conclusión, la experiencia acumulada por el CAT en los tres modelos de integración - aun cuando en este comentario no se consideraron todos sus elementos por el limitado espacio disponible- confirma el valor de iniciativas como ER-SDG en la consolidación de puentes entre el mundo de la investigación sobre implementación de políticas, programas y sistemas de salud, y el mundo de los funcionarios encargados de operar esos programas, servicios e intervenciones relacionadas con la salud. En especial, se reconoce la importancia de acompañar, apoyar y orientar con las acciones necesarias, a fin de garantizar tanto la calidad científica de los resultados de la investigación como la factibilidad de su aplicación para mejorar la implementación de los programas. Paralelamente, se debe subrayar la necesidad de respetar y aprovechar cada contexto, y los arreglos y patrones específicos de relación entre quienes laboran 
en ambos lados, mediante la creación de incentivos para el acercamiento y, de este modo, fortalecer la utilización de los resultados de las investigaciones en la toma de decisiones. Esto, sin dudas, redundará en un mejor desempeño de los sistemas de salud.

Fomentar la participación del personal de salud en la realización de investigaciones con un adecuado rigor científico para orientar la toma de decisiones es un esfuerzo que debe continuar a fin de fortalecer los sistemas de salud de la Región en su camino hacia la cobertura universal de salud y la consecución de los Objetivos de Desarrollo Sostenible.

Contribución de los autores. VBM, PTP y JAR concibieron el estudio original; VBM y PTP escribieron el manuscrito; y LAGB colectó y analizó los datos. Todos los autores revisaron y aprobaron la versión final del manuscrito.

Agradecimientos. A la OPS, la AHPSR y el TDR/OMS por su apoyo en la conformación y desarrollo del CAT, en especial a los doctores Ludovic Reveiz y Vanessa Elías, de la OPS, Étienne V. Langlois, de la AHPSR, y Freddy Pérez, del TDR/OMS, por su permanente seguimiento a los equipos participantes. Se agradece a los participantes de los 13 equipos de investigación por su generosidad al compartir sus experiencias.

\section{Conflictos de intereses. Ninguno a declarar.}

Apoyo financiero. Se contó con el apoyo financiero de la OPS (SCON 2018-00415). Los patrocinadores no participaron de ninguna manera en el diseño del estudio, la colecta y el análisis de los datos, la decisión de publicar este trabajo ni la preparación del manuscrito.

Declaración. Las opiniones expresadas en este artículo son responsabilidad de los autores y no reflejan necesariamente los criterios ni la política de la Revista Panamericana de Salud Pública / Pan American Journal of Public Health y/o de la Organización Panamericana de la Salud.

\section{REFERENCIAS}

1. Organización Panamericana de la Salud. El programa iPIER en las Américas [Internet]. Washington, DC: OPS; 2016 [consultado el 16 de septiembre del 2020]. Disponible en: https://www.paho.org/ hq/index.php?option=com_content\&view $=$ article\&id=11604:improving-program-implementation-through-embedded-research-ipier-program\&Itemid $=41011 \&$ lang $=\mathrm{es}$

2. World Health Organization. Changing mindsets - Strategy on health policy and systems research. Geneva: WHO; 2012.

3. Organización Panamericana de la Salud. Convocatoria: Incorporación de la Investigación para Avanzar en el Cumplimiento de los Objetivos de Desarrollo Sostenible (ER-SDG) [Internet]. Washington, DC: OPS; 2018 [consultado el 16 de septiembre del 2020]. Disponible en: https://www.paho.org/hq/index.php?option=com _content\&view=article\&id=12040: call-for-applications-embedding -research-for-the-sustainable-development-goals\&Itemid $=41749 \&$ lang=es

4. Churruca K, Ludlow K, Taylor N, Long JC, Best S, Braithwaite J. The time has come: Embedded implementation research for health care improvement. J Eval Clin Pract. 2019;25(3):373-80. doi: 10.1111/ jep.13100
5. Langlois EV, Tran NT, Ghaffar A, Reveiz L, Becerra-Posada F. Embedding research in health policy and systems in the Americas. Rev Panam Salud Publica. 2017;41:e68.

6. Peters D, Tran NT. Implementation research in health. A practical guide [Internet]. Geneva: World Health Organization; 2013 [consultado el 16 de septiembre del 2020]. Disponible en: http://r4d.dfid. gov.uk/pdf/outputs/HealthPolicy/implementationguide_eng.pdf

7. Rasanthan K, Tran NT, Johnson H, Hafeez A, Peterson S, Ghaffar A. Realizing the potential of embedded implementation research: Lessons from Pakistan. J Glob Health. 2020;10(2):1-3.

8. Tran N, Langlois EV, Reveiz L, Varallyay I, Elias V, Mancuso A, et al. Embedding research to improve program implementation in Latin America and the Caribbean. Rev Panam Salud Publica. 2017;41:e75.

Manuscrito recibido el 27 de octubre del 2020. Aceptado para publicación, tras revisión, el 31 de diciembre del 2020. 


\section{Embedding research into health services in Latin America and the Caribbean: experiences and challenges of the Technical Support Center}

ABSTRACT This article describes the main models for embedding research and the successful experiences and challenges faced in joint work by researchers and decisionmakers who participated in the Embedding Research for the Sustainable Development Goals (ER-SDG) initiative, and the experience of the Technical Support Center. In June 2018, funding was granted to 13 pre-selected research projects from 11 middle- and low-income countries in Latin America and the Caribbean (Argentina, Bolivia, Brazil, Colombia, Dominican Republic, Ecuador, Guatemala, Guyana, Haiti, Paraguay, and Peru). The projects focused on the system-, policy-, or program-level changes required to improve health and build on the joint work of researchers and decisionmakers, with a view to bringing together evidence production and decision-making in health systems and services. The Technical Support Center supported and guided the production of quality results useful for decision-making. This experience confirmed the value of initiatives such as ER-SDG in consolidating bridges between research on the implementation of health policies, programs, and systems, and the officials responsible for operating health-related programs, services, and interventions. It highlighted the importance of both respecting and taking advantage of each context-and the specific arrangements and patterns in the relationships between researchers and decisionmakers-through incentives for embedded research.

Keywords Implementation science; evidence-informed policy; health services research; Latin America.

\section{Experiências e desafios do Centro de Apoio Técnico na pesquisa integrada aos serviços na América Latina e Caribe}

RESUMO Neste artigo são descritos os principais modelos de integração, as experiências de êxito e os desafios do trabalho conjunto de pesquisadores e responsáveis por tomar decisões que participam da iniciativa Incorporação da Pesquisa para Avançar no Cumprimento dos Objetivos de Desenvolvimento Sustentável (Embedding Research for the Sustainable Development Goals, ER-SDG) e da experiência do Centro de Apoio Técnico (CAT). Em junho de 2018, realizou-se a concessão de financiamento e pré-seleção de 13 projetos de pesquisa provenientes de 11 países de baixa e média renda da América Latina e Caribe (Argentina, Bolívia, Brasil, Colômbia, Equador, Guatemala, Guiana, Haiti, Paraguai, Peru e República Dominicana). Os projetos deveriam enfocar as mudanças necessárias no sistema, políticas ou programas para melhorar a saúde e fundar-se no trabalho conjunto de pesquisadores e responsáveis por tomar decisões visando aproximar a produção de evidências à tomada de decisão nos sistemas e serviços de saúde. O CAT forneceu suporte e orientação à produção de resultados úteis e de qualidade para a tomada de decisão. A experiência confirmou o valor de iniciativas como a ER-SDG para consolidar pontes entre o mundo da pesquisa voltada à implementação de políticas, programas e sistemas de saúde e o mundo dos encarregados de gerir estes programas, serviços e intervenções de saúde. Deve-se enfatizar a necessidade de respeitar e aproveitar cada contexto e os arranjos e padrões próprios da relação entre pesquisadores e responsáveis por tomar decisões criando incentivos à integração.

Palavras-chave Ciência da implementação; política informada por evidências; pesquisa sobre serviços de saúde; América Latina. 